\title{
Taurus Tunable Filter: A Flexible Approach to Narrowband Imaging
}

\author{
Joss Bland-Hawthorn ${ }^{1} \&$ D. Heath Jones ${ }^{2}$ \\ ${ }^{1}$ Anglo-Australian Observatory, PO Box 296, Epping, NSW 2121, Australia \\ jbh@aaossz.aao.gov.au \\ ${ }^{2}$ Mount Stromlo \& Siding Spring Observatories, \\ Private Bag, Weston Creek PO, ACT 2611, Australia \\ Received 1997 August 1, accepted 1997 December 15
}

\begin{abstract}
The Taurus Tunable Filter (TTF) is a tunable narrowband interference filter covering wavelengths from $6300 \AA$ to the sensitivity drop-off of conventional CCDs $(\sim 9600 \AA)$, although a blue 'arm' $(4000-6500 \AA)$ is to be added by the end of 1997. The TTF offers monochromatic imaging at the Cassegrain foci of both the Anglo-Australian and William Herschel Telescopes, with an adjustable passband of between 6 and $60 \AA$. In addition, frequency switching with the TTF can be synchronised with movement of charge (charge shuffling) on the CCD, which has important applications to many astrophysical problems. Here we review the different modes of TTF and suggest their use for follow-up narrowband imaging to the AAO/UKST Galactic Plane $\mathrm{H} \alpha$ Survey.
\end{abstract}

Keywords: instrumentation: detectors - methods: observational — techniques: interferometric

\section{Introduction}

The Taurus Tunable Filter (TTF), manufactured by Queensgate Instruments Pty Ltd, has the appearance of a conventional Fabry-Perot etalon in that it comprises two highly polished glass plates. Unlike conventional Queensgate etalons, however, the TTF also incorporates very large piezoelectric stacks (which determine the plate separation) and highperformance coatings over half the optical wavelength range. The TTF is used in the collimated beam of the TAURUS-2 focal reducer available at both the $3.9 \mathrm{~m}$ Anglo-Australian (AAT) and $4.2 \mathrm{~m}$ William Herschel (WHT) telescopes. Field coverage is 10 arcmin at $\mathrm{f} / 8$ or 5 arcmin at $\mathrm{f} / 15$.

For the first time, TTF provides the capability to synchronise frequency switching with the movement of charge on a CCD, or charge shuffling. This has important benefits for many astrophysical experiments, not least for averaging out temporal variations due to the atmosphere or measurement apparatus. This instrument is an important step in changing the way that intermediate to narrowband imaging is performed at observatories.

The TTF has largely removed the need for buying arbitrary narrow and intermediate interference filters, as one can tune the bandpass and the centroid of the bandpass by selecting the plate spacing. The spacing of the plates is controlled to extremely high accuracy with a capacitance bridge (Jones \& Richards 1973). This approach to tunable imaging has existed since the instrument of Atherton \& Reay (1981), although TTF is the first of its kind in terms of both wavelength and bandpass accessibility. Since tunable filters have periodic transmission profiles, the instrument requires a limited number of blocking filters. At low resolution $(R=300)$, conventional broadband $R$ and $I$ filters suffice. At high resolution ( $R=1000)$, eight intermediate band filters are used to sub-divide $R$ and $I$.

The highly polished plates are coated for optimal performance over the range 6300-9600 $\AA$. The coating reflectivity $(96 \%)$ determines the shape and degree of order separation of the instrumental profile. This is fully specified by the coating finesse $N$, which has a quadratic dependence on the coating reflectivity. The TTF was coated to a finesse specification of $N=40$, which means that the separation between periodic profiles is 40 times the width of the instrumental profile. At such high values, the profile is Lorentzian to a good approximation. For a given wavelength, changes in plate spacing, $L$, correspond to different orders of interference, $m$. This in turn, dictates the resolving power, $R=N m$, according to the finesse.

In the following sections we summarise the different observing modes of TTF. Discussion is also made of phase effects in the field and their influence. The flexibility of TTF will see it well suited to narrowband follow-up from the AAO/UKST Galactic Plane $\mathrm{H} \alpha$ Survey, in lines such as $\mathrm{H} \alpha$, [NII] $\lambda 6583$, [SII] $\lambda 6717$ and $[\mathrm{SII}] \lambda 6731$. 
We maintain a WWW site at

$$
\text { http : //msowww.anu.edu.au/ dhj/ttf.html }
$$

describing all aspects of TTF and its operation. In addition to general use, the instrument is available for AAT service time at

http : //www.aao.gov.au/local/www/jmc/service/service.html

if observations are shorter than 3 hours.

\section{CCD Charge Shuffling}

Central to almost all modes of TTF use is charge shuffling. Charge shuffling is the movement of charge along the CCD between multiple exposures of the same frame, before the image is read out (Clemens \& Leach 1987; Cuillandre et al. 1994). An aperture mask ensures that only one section of the CCD frame is exposed at a time. For each exposure, the tunable filter is systematically moved to different gap spacings in a process called frequency switching. In this way, a region of sky can be captured at several different wavelengths on the one image (Figure 1). Alternatively, the TTF can be kept at fixed frequency and charge shuffling performed to produce time-series exposures. Each of these modes is described in the following section.

The TTF plates can be switched anywhere over the physical range $2-12 \mu \mathrm{m}$ at rates in excess of $100 \mathrm{~Hz}$, although in most applications, these rates rarely exceed $0 \cdot 1 \mathrm{~Hz}$. If a shutter is used, this limits the switching rate to about $1 \mathrm{~Hz}$. Charge on a large-format CCD can be moved over the full area at rates approaching $10 \mathrm{~Hz}$ : it is only when the charge is read out through the amplifiers that this rate is greatly slowed down. The TTF exploits the ability of certain large-format CCDs to move charge up and down many times before significant signal degradation occurs (Yang et al. 1989). In this way, it is possible to form discrete images taken at different frequencies where each area of the detector may have been shuffled into view many times to average out temporal effects.

The field of view available in shuffle mode depends on the number of frequencies being observed. When we move the charge upwards, say, information in pixels at the top of the field is rolled off the top and lost. For example, two frequencies requires that we divide the CCD into three vertical partitions where information in one of the outer partitions is lost in the shuffle process. In the limit of $n$ frequencies, where $n$ is large, only half the available detector area is used to store information. The new MIT-LL $4096 \times 2048$ (rows $\times$ columns) format CCDs with $15 \mu \mathrm{m}$ pixels will increase the detector area available for shuffling by threefold compared to the present Tek $1024 \times 1024$ CCD $(24 \mu \mathrm{m}$ pixels $)$. This is because the instrument field of view projects to an aperture 1024 pixels in diameter and shuffling is only possible in the vertical direction.

One application of charge shuffling that does not sacrifice detector area is time-series imaging (Section $3 \cdot 3$ ). This is because the imaging region is relatively small and able to be read out as the time series progresses.

\section{Observing Modes of the TTF}

There are several technical problems driving the development of tunable filters for narrowband imaging over the use of standard fixed interference filters. First, it is difficult and very expensive for manufacturers to produce high-performance narrow passbands,

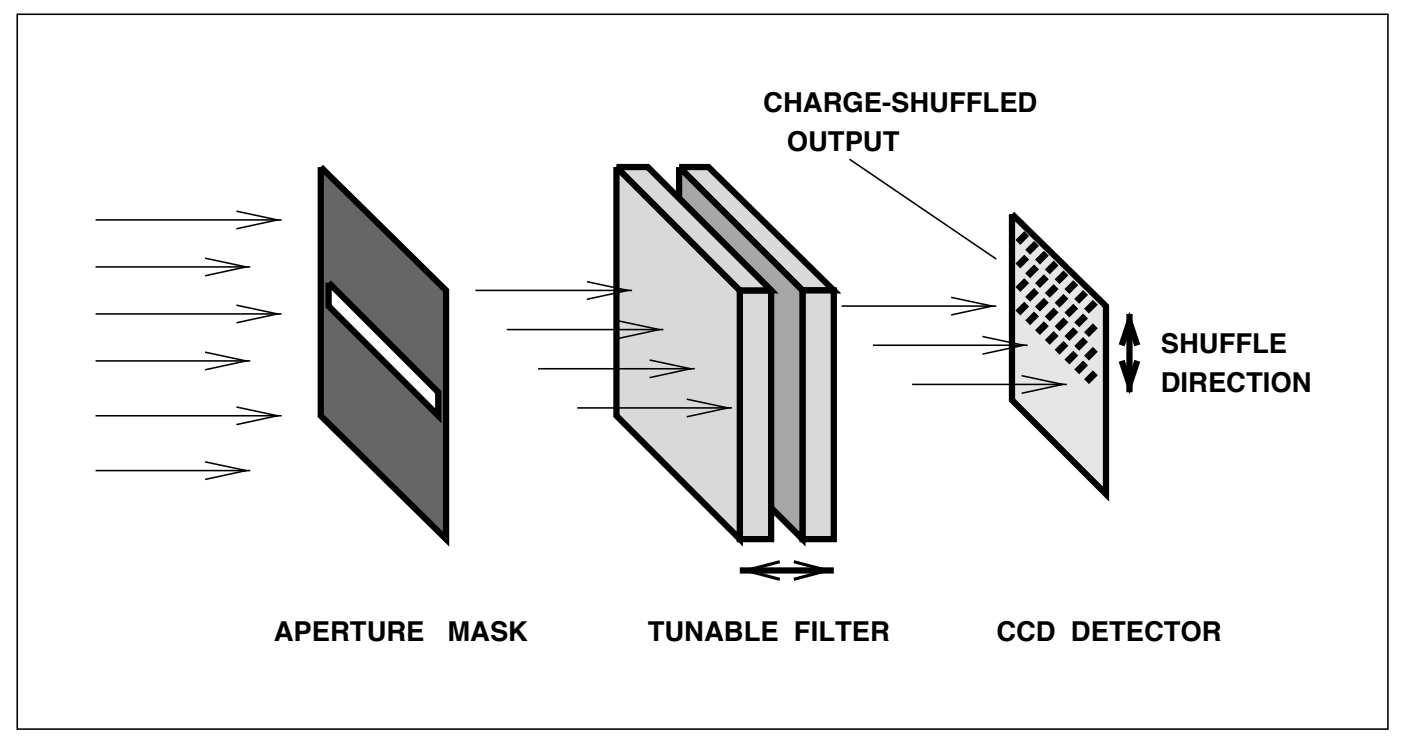

Figure 1-Simplified view of how the CCD chip is illuminated and shuffled. The mask aperture size controls the amount of the CCD exposed and therefore the number of different images fitted onto an individual frame. Charge can be shuffled both up and down the chip. 
particularly at resolving powers approaching 1000 . This problem is largely circumvented by the use of TTF in conjunction with a five-cavity, intermediate bandpass blocking filter. Second, the TTF instrumental function has identical form at all wavelengths and all bandpasses. A comparison of two discrete bands at different wavelengths is moderated only by the blocking filter transmission at those wavelengths (which is normally flat in any case), and the ratio of the gap spacings. Third, the same optical path is used at all frequencies. Furthermore, the ability to shuffle charge allows one to average out all temporal variations: atmospheric transparency, the contribution from atmospheric lines, seeing, detector and electronic instabilities.

We now describe some of the advantages to be had from the TTF shuffle capability in imaging emission-line sources.

\subsection{Tuning to a Specific Wavelength and Bandpass}

This allows us to obtain images of obscure spectral lines at arbitrary redshifts (see e.g. Jones \& BlandHawthorn 1997a). We can also optimise the bandpass to accomodate the line dispersion and to suppress the sky background. The off-band frequency can be chosen to avoid night-sky lines and can be much wider so that only a fraction of the time is spent on the off-band image. Figure $2 \mathrm{a}$ shows a charge-shuffled image of the planetary nebula NGC 2438 in [SII] lines at 6731 and $6717 \AA$. During this 12 min exposure, TTF was switched between the two frequencies 18 times while the charge was shuffled back and forth accordingly. In this way, temporal variations in atmospheric transmission are equally shared between each passband over the entire exposure time. (a)

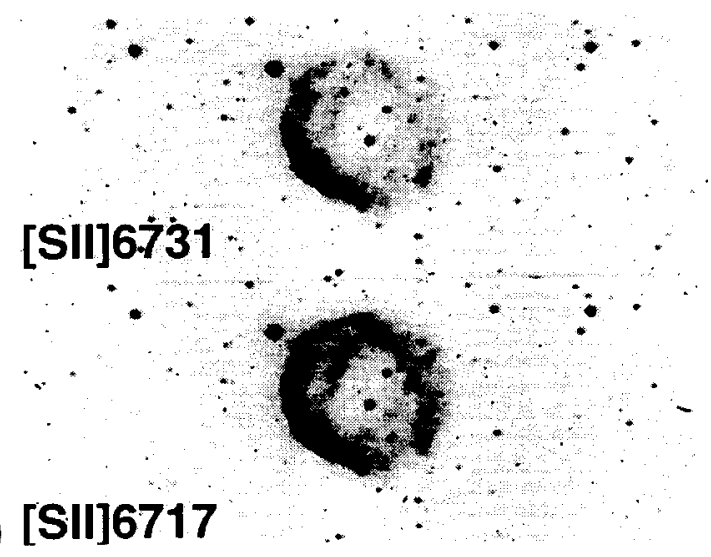

(b)

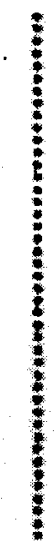

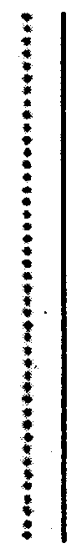

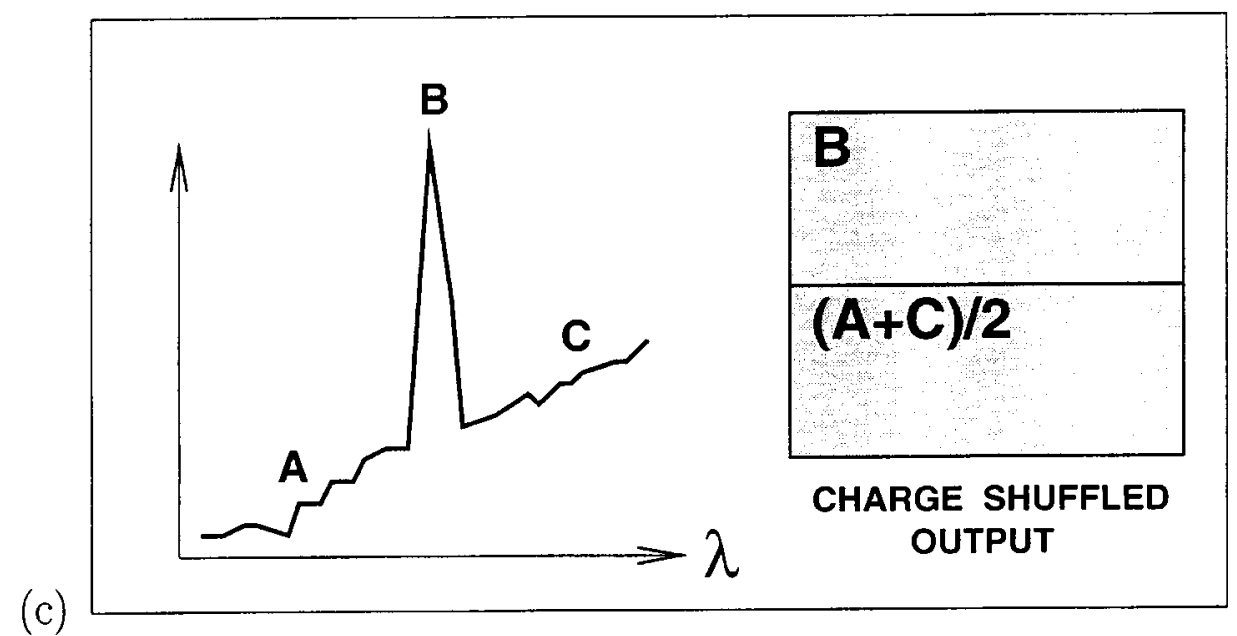

Figure 2-(a) Charge-shuffled image of the planetary nebula NGC 2438 in [SII] $\lambda 6371$ (top) and [SII] 6717 (bottom). The TTF was frequency-switched 18 times during the $12 \mathrm{~min}$ exposure and the charge shuffled back and forth in concert. A $10 \AA$ bandpass was used. (b) Charge-shuffle time-series imaging of V2116 Oph, an X-ray pulsar in orbit around a red giant. V2116 Oph is to the right, reference stars are at the centre and left. TTF was tuned to a $7 \AA$ bandpass centred on the [OI] $\lambda 8446$ line, which is suspected to vary with the pulsar period of $120 \mathrm{~s}$. Each exposure was $12 \mathrm{~s}$. (c) Charge shuffling either side of an emission-line to average out the effect of a steeply sloping continuum. Equal time is spent collecting continuum from both sides of the emission-line (left). The line only contributes to the top portion of the shuffle frame, while both sides of the continuum contribute to the bottom (right). 


\subsection{Shuffling between On and Off-band Frequencies}

With the new MIT-LL chips, we are able to image the full 10 arcmin field for two discrete frequencies. We can also choose a narrow bandpass for the on-band line and a much broader bandpass (factor of 4-5) for the off-band image so that we incur only a $20 \%$ overhead for the off-band image. As with specific tuning (Section 3.1), multiple frequencies can be imaged in a single frame, the number of which is entirely arbitrary.

\subsection{Time-series Imaging}

For time-varying sources, we can step the charge in one direction while only switching between a line and a reference frequency. For example, a compact variable source imaged through a narrow aperture in the focal plane forms a narrow image at the detector. We can switch between the line and a reference frequency many times, forming a set of narrow interleaved images at the detector. The reference frequency is a measure of the atmospheric stability during the time series. Alternatively, if nearby reference stars are available for photometric calibration, then charge shuffling can be done at a single fixed frequency as demonstrated in Figure $2 \mathrm{~b}$. For example, some X-ray binaries produce strong emission lines that vary on $0 \cdot 1 \mathrm{~Hz}$ timescales. With a slit only 4 pixels wide, we can obtain 500 images in the emission line, interleaved with a further 500 images at a reference frequency. In this example, all of the CCD area is utilised because the charge is clocked in only one direction. The vertical shift takes about $50 \mu \mathrm{s}$ per row. Strictly speaking, this operation constitutes a 'charge shift' rather than a charge shuffle. Once the chip is full, it takes $\sim 3 \mathrm{~min}$ to read out the CCD.

If the readout time of several minutes is critical to the measurement, an alternative method is timeseries readout. For the example above, the four CCD rows comprising the slit are clocked downwards at the end of each exposure. However, the next exposure is delayed by the time it takes to read out the bottom four rows $(\sim 200 \mathrm{~ms})$. In practice, the slow shutter means that the time-series mode is to be preferred for most applications over the 'charge-shift' mode.

\subsection{Adaptive Frequency Switching with Charge Shuffling}

When imaging spectral lines that fall between $\mathrm{OH}$ bandheads or on steeply rising continua, a powerful feature is the ability to shuffle between on and off bands, but where the off-band is alternated between two or more frequencies either side of the on-band frequency. As illustrated in Figure 2, this technique can be used to average out either rapid variations in blocking filters or the underlying spectral continuum.
3.5 Shuffling Charge in One Direction with a Narrow Focal-plane Slit

This allows us to produce a long-slit spectrum of an extended source. While this is vastly less efficient than using a long-slit spectrograph, the capability is a fundamental component of establishing the parallelism of reflecting mirrors at few-micron spacings (Jones \& Bland-Hawthorn 1997b). We do this by taking long-slit TTF spectra of an arc line, with a pupil-plane mask to isolate a particular section of the beam. The plates are adjusted until the line shows peak transmission at a common plate setting for all regions of the beam. It is then that the plates are parallel. Conventional methods are an order of magnitude slower by comparison.

\section{Phase Effects}

The primary goal of a tunable filter is to provide a monochromatic field over as large a detector area as possible. With the present TTF, however, the field of view is not strictly monochromatic. The effect is most acute at high orders of interference. Figure $3 \mathrm{a}$ shows how wavelength gradients (or phase effects) are evident from a ring pattern of atmospheric $\mathrm{OH}$ emission lines across the TTF field. In this particular case we see rings at different wavelengths appearing within the one order. The circular pattern is not centrally aligned, due to tilting of the plates $\left(\sim 10\right.$ to $\left.15^{\circ}\right)$ to deflect ghost images from the beam.

Wavelengths are longest at the centre and get bluer the further one moves off-axis. For instruments such as TTF, the wavelength as a function of off-axis angle $\theta$ is ${ }^{1}$

$$
\lambda(\theta)=\lambda_{\text {centre }}\left(1-\frac{\theta^{2}}{2}\right) .
$$

Here $\lambda_{\text {centre }}$ is the on-axis wavelength, equal to $2 L / m$ (for an air gap), where $L$ is the physical plate spacing and $m$ is the order of interference.

It follows from equation (1) that the change in wavelength $\lambda_{\phi}$ across an angle $\theta$ from the centre is given by

$$
\lambda_{\phi}(\theta)=\lambda_{\text {centre }}\left(\frac{\theta^{2}}{2}\right) .
$$

Since $\theta \lesssim 0 \cdot 14$ for the current Tektronix CCD, then $\lambda_{\phi} / \lambda_{\text {centre }}$ is always $\lesssim 2 \cdot 5 \times 10^{-3}$ when the TTF is used on-axis, although $\lesssim 10^{-2}$ when the centre of the ring pattern is tilted to the edge of the field. It also follows that $\lambda_{\phi} / \lambda_{\text {centre }}$ remains fixed over a given radius, irrespective of order $m$. Bland \& Tully

\footnotetext{
${ }^{1}$ Equation (1) makes use of the small-angle approximation for $\cos \theta$.
} 


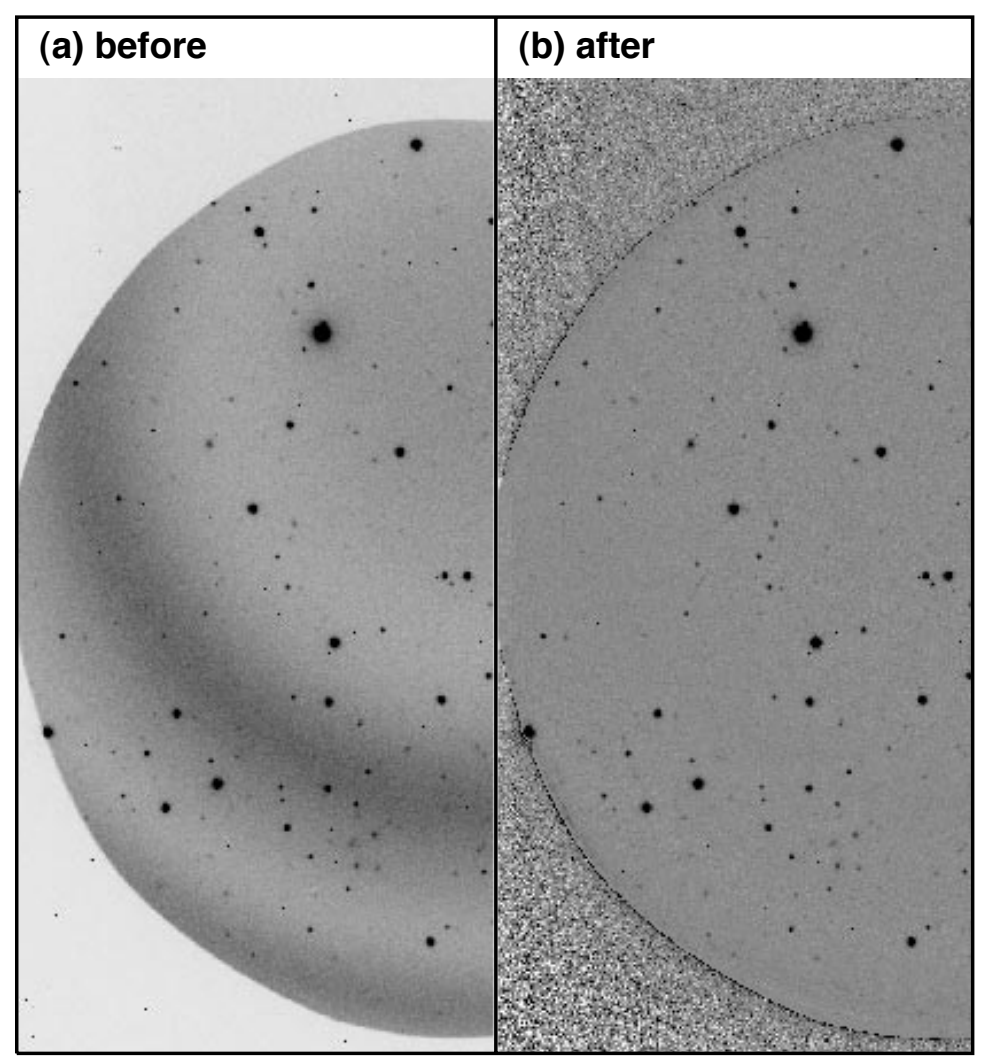

Figure 3-Images showing the removal of the atmospheric $\mathrm{OH}$ emission lines from a raw TTF image at high spectral resolution (a) before and (b) after cleaning. Only half the full TTF field is shown in each case.

(1989) derived related equations in the context of a higher-order conventional etalon [in sections $\mathrm{II}(c)$, (e)].

We define our monochromatic field by the size of the Jacquinot spot, the central region of the ring pattern. By definition, the Jacquinot spot is the region over which the wavelength changes by no more than $\sqrt{2}$ of the etalon bandpass, $\delta \lambda$. For wavelength $\lambda$ the bandpass relates directly to the order $m$ such that

$$
\delta \lambda=\lambda / N m=\lambda /(40 \times m) .
$$

Here $N$ is the effective finesse of the etalon, which in the case of TTF is approximately 40. Combining equations (1) and (3), we find that the angle subtended by the Jacquinot spot is

$$
\theta_{\mathrm{Jac}}{ }^{2}=2 \sqrt{2} / N m=1 /(14 \cdot 1 \times m) .
$$

For a particular etalon, the size of the Jacquinot spot depends on order $m$ alone. Equation (4) shows how the spot covers increasingly larger areas on the detector as the filter is used at lower orders of interference. The absolute wavelength change across the detector remains the same, independently of order. However, its effect relative to the bandpass diminishes as $m$ decreases.

Figure $3 \mathrm{~b}$ demonstrates how the effects of atmospheric emission can be removed during reduction.
The software creates a background map by medianfiltering copies of the original image, each one offset from the other by a small amount. The result is smoothed and subtracted from the original, leaving little or no night-sky residual. This technique relies on the fact that at low orders of interference, the night sky rings are lower-frequency structures than the objects.

The TTF is the most straightforward application of tunable filter technology. Other, more sophisticated techniques such as acousto-optic filters exist (see Bland-Hawthorn \& Cecil 1996 for a review), although all are currently considerably more expensive. In future TTF-type instruments, phase effects will be eliminated from the outset by bowed plates. One advantage of such a design is that the TTF will no longer have to be tilted to deflect ghost reflections. Furthermore, interference coatings are notorious for bowing plates and this can be factored into the plate curvature specification. Other possible improvements are additional cavities to square up the instrument profile. All of these modifications are currently being explored.

\section{Summary}

We have discussed details of the Taurus Tunable Filter (TTF) instrument and its use. When used in conjunction with a CCD charge-shuffling technique, the TTF is well suited for multi-narrowband imaging 
of emission-line sources. As such, it is ideal for follow-up imaging to the AAO/UKST Galactic Plane $\mathrm{H} \alpha$ Survey, for sources that are $\sim 10$ arcmin or smaller. The analysis of tunable filter data is more straightforward than traditional Fabry-Perot imaging, as all regions of the field are close to a common wavelength.

The present TTF instrument exhibits some phase effects at high resolving powers. We have demonstrated that these effects are tolerable for most applications $\left(\lambda_{\phi} / \lambda_{\text {centre }} \lesssim(2 \cdot 5-10) \times 10^{-2}\right)$ and the side-effects correctable through software. The commissioning of TTF has marked the start of an exciting period of new imaging instruments for optical astronomy.

\section{Acknowledgments}

Thanks to J. R. Barton, L. G. Waller, T. J. Farrell, E. J. Penny and C. McCowage for technical input during the TTF and charge-shuffle implementation. DHJ acknowledges the assistance of a Commonwealth Australian Postgraduate Research Award.

Atherton, P. D., \& Reay, N. K. 1981, MNRAS, 197, 507 Bland, J., \& Tully, R. B. 1989, AJ, 98, 723

Bland-Hawthorn, J., \& Cecil, G. N. 1996, in Atomic, Molecular and Optical Physics: Atoms and Molecules, Vol. 29B, ch. 18, ed. F. B. Dunning \& R. G. Hulet (New York: Academic)

Clemens, D. P., \& Leach, R. W. 1987, Opt. Eng., 26, 9 Cuillandre, J. C., et al. 1994, A\&A, 281, 603

Jones, D. H., \& Bland-Hawthorn, J. 1997a, PASA, 14, 8

Jones, D. H., \& Bland-Hawthorn, J. 1997b, PASP, submitted Jones, R. V., \& Richards, J. C. S. 1973, J. Phys. E, 6, 589 Yang, F. H., Blouke, M. M., Heidtmann, D. L., Corrie, B., Riley, L. D., \& Marsh, H. H. 1989, in Optical Sensors and Electronic Photography (Proc. SPIE), ed. M. M. Blouke \& D. Pophal (Washington: SPIE-International Society for Optical Engineering), p. 213 